CrossMark \& click for updates

Cite this: DOI: $10.1039 / \mathrm{c5}$ cp04958j

\title{
Crystallization of undercooled liquid fenofibrate $\dagger$
}

\begin{abstract}
Esther Amstad, ${ }^{\mathrm{ab}}$ Frans Spaepen ${ }^{\mathrm{a}}$ and David A. Weitz*ac
Formulation of hydrophobic drugs as amorphous materials is highly advantageous as this increases their solubility in water and therefore their bioavailability. However, many drugs have a high propensity to crystallize during production and storage, limiting the usefulness of amorphous drugs. We study the crystallization of undercooled liquid fenofibrate, a model hydrophobic drug. Nucleation is the ratelimiting step; once seeded with a fenofibrate crystal, the crystal rapidly grows by consuming the undercooled liquid fenofibrate. Crystal growth is limited by the incorporation of molecules into its surface. As nucleation and growth both entail incorporation of molecules into the surface, this process likely also limits the formation of nuclei and thus the crystallization of undercooled liquid fenofibrate, contributing to the good stability of undercooled liquid fenofibrate against crystallization.
\end{abstract}

Received 20th August 2015,

Accepted 15th October 2015

DOI: $10.1039 / c 5 c p 04958 j$

www.rsc.org/pccp produced using a microfluidic nebulator. ${ }^{8}$ However, studies on the crystallization of pure amorphous drugs remain rare.

In this paper, we study the crystallization of undercooled liquid fenofibrate, a hydrophobic model drug. Even though its crystallization is limited by nucleation, both the nucleation and growth processes entail the incorporation of solute molecules into crystalline surfaces; hence, their rates are influenced by similar kinetic parameters. We study crystal growth since this process is easier to visualize; it is diffusion-limited and thus strongly temperature-dependent. However, diffusion only accounts for the frequency with which molecules impinge on a surface; it does not account for the probability of impinging molecules to insert themselves into the crystal. This probability is very low for fenofibrate, an anisotropic molecule, and slows down the crystal growth by more than two orders of magnitude under the conditions of these experiments.

\section{Results and discussion}

We fabricate the nebulator from poly(dimethyl siloxane) (PDMS) using soft lithography. ${ }^{9}$ It contains two inlets for liquids and six inlets for air. Liquid is injected into the main channel before the first pair of air inlets intersects the main channel at an angle of $135^{\circ}$. The air pushes ethanol towards the four channel walls, forming thin ethanol films that flow along these walls while the air flows through the center of the channel. The subsequent air inlets intersect the main channel at an angle of $45^{\circ}$, as shown in Fig. S1 (ESI $\dagger$ ); the air inserted through these inlets accelerates the air in the main channel, thereby thinning the ethanol films. The last junction is three-dimensional (3D) ${ }^{10}$ to push the ethanol films away from all four walls of the last part of the main channel, thereby breaking the ethanol films into very small drops that rapidly dry. ${ }^{8}$ 
We use fenofibrate as a hydrophobic model drug; amorphous fenofibrate is an undercooled liquid at room temperature since it has a glass transition temperature, $T_{\mathrm{g}}$, of $-20{ }^{\circ} \mathrm{C}^{7}$ and a melting temperature, $T_{\mathrm{m}}$, of $80{ }^{\circ} \mathrm{C} .{ }^{11}$ We dissolve fenofibrate in ethanol at $5 \mathrm{mg} \mathrm{ml}^{-1}$, and inject the solution through one liquid inlet at $1 \mathrm{ml} \mathrm{h}^{-1}$ using syringe pumps; we block the second liquid inlet to avoid leakage of the liquid and the air. We introduce air by applying 0.28 MPa to all the air inlets and collect the spray dried undercooled liquid drops $15 \mathrm{~cm}$ apart from the nozzle on a flat substrate. ${ }^{8}$

The nebulator produces amorphous undercooled liquid fenofibrate drops with diameters as small as $14 \mathrm{~nm} ;{ }^{12}$ the volume of a single drop is as low as $10^{-21} \mathrm{l}$. However, as amorphous fenofibrate is an undercooled liquid at room temperature, drops coalesce as soon as they come in contact with each other. The larger volume of the resulting coalesced drops increases the probability for a crystal nucleus to form in each drop. ${ }^{13}$ Remarkably, even if we coalesce sufficient drops to form a drop as large as $600 \mathrm{pl}$, fenofibrate remains amorphous for at least 5 days if stored at room temperature under ambient conditions. However, once seeded with a crystal nucleus of a diameter between $35 \mu \mathrm{m}$ and $70 \mu \mathrm{m}$, three dimensional crystals grow very rapidly by consuming some of the amorphous phase; it takes less than a minute to grow dendritic crystals several $100 \mu \mathrm{m}$ in size, as shown in time-lapse optical micrographs in Fig. 1a-d, indicating that nucleation is the rate-limiting step in the crystallization process.
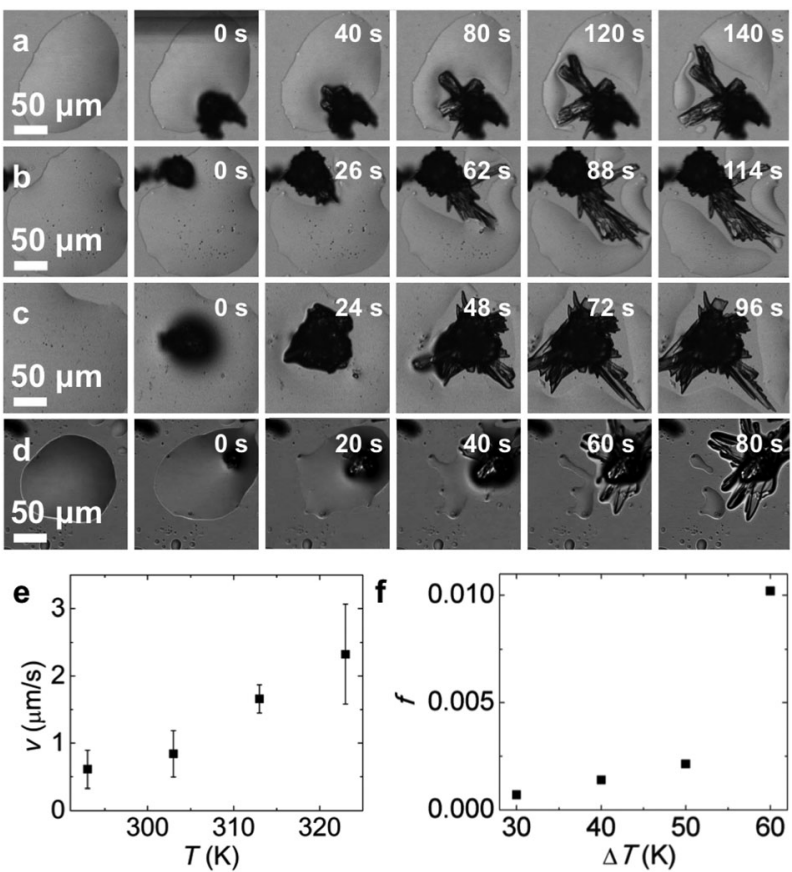

Fig. 1 ( $a-d)$ Time-lapse confocal microscopy images of undercooled liquid fenofibrate seeded with a fenofibrate crystal at (a) $293 \mathrm{~K}$, (b) $303 \mathrm{~K}$, (c) $313 \mathrm{~K}$, and (d) $323 \mathrm{~K}$. The time after contact of the crystalline nucleus with the undercooled liquid fenofibrate drop is indicated in the insets. (e) The maximum growth rate of the dendrite tip as a function of the temperature at which the undercooled liquid is crystallized. (f) The probability for fenofibrate molecules to insert themselves into the surface, $f$, is shown as a function of the undercooling, $\Delta T$.
The formation of crystal nuclei is difficult to study quantitatively. However, nucleation involves the incorporation of solute molecules into a crystal surface; the same process also occurs during crystal growth, which is much easier to access experimentally. Thus, we estimate the temperature-dependent rate at which molecules are incorporated into the crystal surface by quantifying the growth kinetics of the dendrite tips. We measure the distance dendrite tips grow per unit as a function of the temperature, $T$, using time-lapse optical micrographs and calculate the dendrite growth velocity. During the early and intermediate stages of the crystal growth the growth rate is, within experimental error, constant. By contrast, the growth rate continuously decreases during the final stages of crystal growth. Hence, we only quantify the growth rate during the early and intermediate stages. As we increase the temperature, the undercooling $\Delta T=T_{\mathrm{m}}-T$ decreases; here $T_{\mathrm{m}}$ is the melting temperature of fenofibrate; this reduces the driving force for crystal growth. ${ }^{13}$ Despite the reduced driving force, crystals grow faster with increasing temperature, as shown in Fig. 1e.

The increasing growth rate of crystals with increasing temperature indicates that crystal growth is diffusion-limited. Factors limiting the growth rate of fenofibrate crystals must thus include the jump frequency of the molecule from the liquid to the crystal, $k$, and the probability that impacting molecules are incorporated into the surface, which is described by the site factor, $f$. The diffusional jump frequency $k=\frac{6 D}{\lambda^{2}}$ depends on the diffusion coefficient, $D$, as well as the jump distance, $\lambda$, which we take as the longest dimension of a fenofibrate molecule, $1.5 \mathrm{~nm} ;{ }^{14}$ it is independent of the mechanism by which crystals grow.

To investigate the growth mechanism, we determine the temperature-dependence of $f$. We describe the growth velocity as $v=k f \lambda\left(1-\exp \left(-\frac{\Delta \mu}{k_{\mathrm{B}} T}\right)\right)$; here, $\Delta \mu$ is the difference in the chemical potential of a molecule in the crystal and in the undercooled liquid, $k_{\mathrm{B}}$ the Boltzmann constant, and $T$ the absolute temperature. We calculate $\Delta \mu=\frac{\Delta H_{\mathrm{m}} \Delta T}{T_{\mathrm{m}} N_{\mathrm{Av}}}$ as a function of the undercooling using the latent heat of fenofibrate, $\Delta H_{\mathrm{m}}=$ $28.8 \mathrm{~kJ} \mathrm{~mol}^{-1}$, and its melting temperature, $T_{\mathrm{m}}=80{ }^{\circ} \mathrm{C}$, which we determine with differential scanning calorimetry (DSC) (Fig. 2); here $N_{\mathrm{Av}}$ is Avogadro's number. In addition, we quantify the diffusional jump frequency $k=\frac{6 D}{\lambda^{2}}=\frac{2 k_{\mathrm{B}} T}{\pi \eta \lambda^{3}}$ using the StokesEinstein relation for the last equality and the temperaturedependent values for the viscosity of fenofibrate, $\eta \cdot{ }^{4}$ We subsequently calculate $f$ as a function of temperature. At room temperature, the site factor is as low as 0.01 and further decreases with increasing temperature, as shown in Fig. 1f. These small values slow down crystal growth. Moreover, the increase in $f$ with increasing undercooling, $\Delta T$, suggests that the crystal growth is limited by the layer nucleation on the crystal face, as detailed in the ESI. $\dagger$

The key parameter in the nucleation of a crystal is the ledge energy, $\gamma_{\text {le }}$, associated with the side surface of the layer nucleus. ${ }^{13}$ 


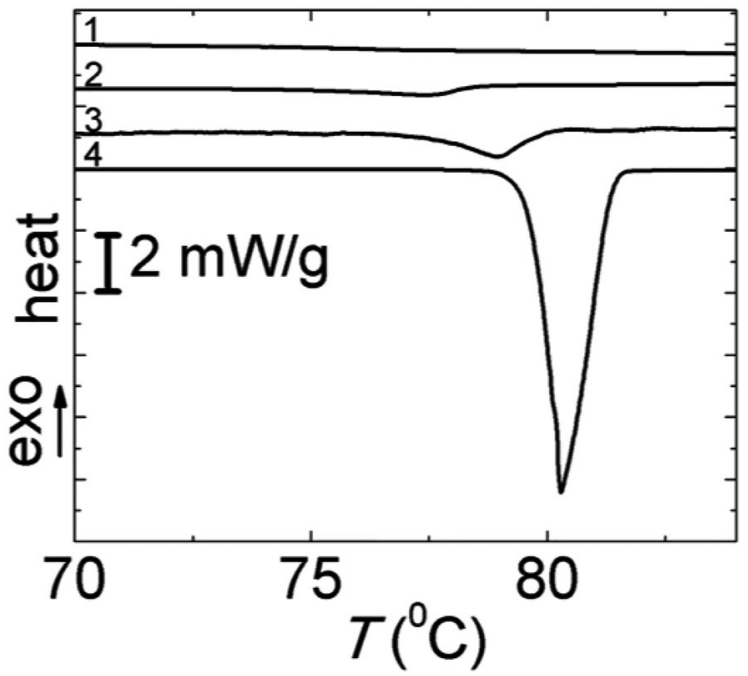

Fig. 2 Differential scanning calorimetry (DSC) traces of spray dried undercooled liquid fenofibrate drops with a diameter of $14 \mathrm{~nm}$. Traces were acquired (1) $0 \mathrm{~d}$, (2) $4 \mathrm{~d}$, and (3) $8 \mathrm{~d}$ after preparation. Samples are stored in an aluminum DSC pan under ambient conditions. (4) Reference spectrum of bulk fenofibrate.

To compute $\gamma_{\text {le }}$, we express the site factor as $f=\left(\lambda^{2} n_{\mathrm{s}}{ }^{*} \frac{1}{3} f_{1}^{\frac{2}{3}} i_{\mathrm{s}}{ }^{*} \frac{1}{3}\right.$; here $n_{\mathrm{s}}^{*}$ is the surface concentration of critical layer nuclei, $f_{1}$ is the site factor for the ledge of a layer, and $i_{\mathrm{s}}{ }^{*}$ the number of molecules along the edge of a critical layer nucleus. The site factor for a ledge is typically much larger than that for a surface since ledges are usually rough; ${ }^{15}$ we thus take $f_{1} \approx 1$. The number of molecules in a critical layer nucleus is small and we approximate $i_{\mathrm{s}}{ }^{*} \approx 1$. We confirm the accuracy of this assumption by determining the size of the critical nucleus after we have calculated the ledge energy. The surface concentration of critical nuclei, $n_{\mathrm{s}}{ }^{*}=\frac{1}{\lambda^{2}} \exp \left(-\frac{w_{\mathrm{s}}{ }^{*}}{k_{\mathrm{B}} T}\right)$, depends on the work for these critical nuclei to form, $w_{\mathrm{s}}{ }^{*}=\pi \frac{\gamma_{\mathrm{le}}{ }^{2} \lambda}{\Delta \mu_{\mathrm{v}}}$. This work is a balance between the energy per unit length required to form a ledge, $\gamma_{\text {le }}$, and the energy gained by forming a crystal. The gained energy is equal to the difference in the chemical potential per unit volume, $\Delta \mu_{\mathrm{v}}=\frac{\Delta \mu}{\bar{V}}$; here $\bar{V}$ is the molecular volume of fenofibrate. Thus, we approximate the interfacial site factor as $f=\exp \left(-\frac{w_{\mathrm{s}}{ }^{*}}{3 k_{\mathrm{B}} T}\right)$. Using the values of $f$, shown in Fig. 1f, we calculate the ledge energy to be $\gamma_{\mathrm{le}}=14 \mathrm{~mJ} \mathrm{~m}^{-2}$. Indeed, this value is nearly independent of the temperature, despite the temperature-dependence of $f$ and $\Delta \mu_{\mathrm{v}}$, as shown in the ESI, $\dagger$ supporting the validity of the layer nucleation mechanism.

As crystallization of fenofibrate is limited by the nucleation of a crystal, the stability of amorphous fenofibrate strongly depends on the rate at which stable crystal nuclei form. We experimentally determine an upper limit of the crystal nucleation rate, $I<\frac{1}{V_{\mathrm{d}} \Delta t}$; here $V_{\mathrm{d}}$ is the volume of a drop and $\Delta t$ the time it takes for a drop to crystallize. We spray dry undercooled liquid fenofibrate drops with different volumes and measure the time it takes them to crystallize. Well-separated drops as small as $10^{-21} \mathrm{l}$ remain liquid for more than 7 months if stored at room temperature. ${ }^{8}$ If we increase the volume of the drops to 0.1-1 pl, they show no sign of crystallinity after 1 day storage in an aluminum DSC pan, as indicated by the absence of any melting peaks in DSC trace 1 of Fig. 2. However, they do start to crystallize within 4 days, as indicated by the melting peak in trace 2 of Fig. 2. Even more of these drops spontaneously crystallize after having been stored for 8 days, as indicated by the larger melting peak in trace 3 in Fig. 2. If we increase the volume of drops by 11 orders of magnitude to $V_{\mathrm{d}} \approx 600 \mathrm{pl}$, they only remain liquid for approximately $\Delta t=5$ days. Thus, we obtain $I<\frac{1}{V_{\mathrm{d}} \Delta t} \approx 3.8 \times 10^{6} \mathrm{~m}^{-3} \mathrm{~s}^{-1}$ at room temperature.

A crystal nucleus becomes supercritical and thus stable when the energy gained from adding an additional molecule $\Delta \mu$, is higher than the energy needed to create additional crystal-melt interface; the energy of this additional crystalmelt interface per unit area is $\gamma$. For a spherical nucleus, the critical radius is $r^{*}=\frac{2 \gamma}{\Delta \mu_{\mathrm{v}}}$. To determine $r^{*}$, we use the classical nucleation theory to describe the nucleation rate $I=f k n Z \exp \left(-\frac{w^{*}}{k_{\mathrm{B}} T}\right)$; here, $n$ is the number of molecules per unit volume, $Z$ the Zeldovich factor, and $w^{*}=\frac{16 \pi}{3} \frac{\gamma^{3}}{\left(\Delta \mu_{\mathrm{v}}\right)^{2}}$ the work to form a spherical nucleus. ${ }^{13}$ From the density of fenofibrate, we get $n=2 \times 10^{27} \mathrm{~m}^{3} \mathrm{~s}^{-1}$. Taking the lower limit of $Z \geq$ $0.01,{ }^{13}$ the measured values for $f$ and $\Delta \mu_{\mathrm{v}}$, and the calculated values for $k$, we estimate a lower limit for $\gamma \geq 15 \mathrm{~mJ} \mathrm{~m}^{-2}$; this value is close to that of the ledge energy $\gamma_{l e}$. At room temperature, this surface energy results in a radius of a critical nucleus
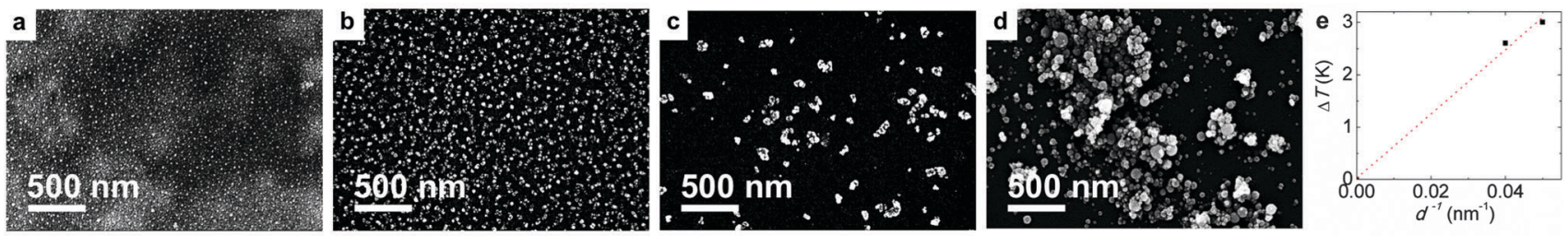

Fig. 3 (a-d) Scanning electron micrographs of undercooled liquid fenofibrate drops spray dried by applying (a) $0.28 \mathrm{MPa}$, (b) $0.24 \mathrm{MPa}$, (c) $0.21 \mathrm{MPa}$, and (d) $0.17 \mathrm{MPa}$ to the inlets of the microfluidic nebulator. (e) Size-dependent melting point depression of fenofibrate. The slope of the linear fit through the origin allows calculation of $\Delta \gamma$, the difference of the surface energies between the free surfaces at the liquid and crystalline phase. 
of approximately $1.8 \mathrm{~nm}$; such a nucleus contains approximately 26 fenofibrate molecules. ${ }^{14}$ Despite of the small size of the critical crystal nucleus, it takes time for them for such nuclei to form, as the probability for fenofibrate molecules to incorporate in a crystal, $f$, is very low.

Once the crystal growth is complete, a particle of radius $r_{\mathrm{p}}$ is formed whose chemical potential differs from that of the bulk by $\Delta \mu=\frac{2 \gamma_{\mathrm{c}} \bar{V}}{r}$; here $\gamma_{\mathrm{c}}$ is the crystal free energy. Since the surface energy of the liquid, $\gamma_{1}$, is usually lower than that of the crystal, $\gamma_{c}$, the melting temperature is shifted, according to the Gibbs-Thompson relation $\Delta T=T_{\mathrm{m}, \mathrm{b}}-T_{\mathrm{m}, \mathrm{p}}=\frac{2 \Delta \gamma \bar{V} T_{\mathrm{m}}}{r \Delta H_{\mathrm{m}}}$; here $T_{\mathrm{m}, \mathrm{b}}$ is the melting point of the bulk, $T_{\mathrm{m}, \mathrm{p}}$ that of the particle, and $\Delta \gamma=\gamma_{\mathrm{c}}-\gamma_{1}$. We spray dry particles with a narrow size distribution, having diameters between $20 \mathrm{~nm}$ and $25 \mathrm{~nm}$ by controlling the pressure applied to the air inlets of the nebulator. ${ }^{8}$ We measure their sizes from scanning electron microscopy (SEM) images, as shown in Fig. 3a-d, quantify $\Delta T$ using differential scanning calorimetry (DSC), ${ }^{8}$ and plot $\Delta T$ as a function of the inverse particle diameter. Indeed, the melting point depression increases with decreasing particle size, as shown in Fig. 3e. We perform a linear fit of the data through the origin to obtain the difference between the surface energy of the crystal and the liquid $\Delta \gamma=4.5 \mathrm{~mJ} \mathrm{~m}^{-2}$, a plausible value.

\section{Conclusions}

The crystallization of undercooled liquid fenofibrate is limited by nucleation. Even though we only show that the incorporation probability of molecules arriving at the surface is the ratelimiting step of the crystal growth, we expect the same parameter also to limit the nucleation as both processes, nucleation and growth, entail the incorporation of molecules into a crystal surface. Thus, the low insertion probability of fenofibrate molecules into the crystal slows down the nucleation of crystals, thereby enhancing the stability of the undercooled liquid. This is a contributing reason why undercooled liquid fenofibrate does not instantaneously crystallize and, if compartmentalized into small drops, displays an unprecedented stability against crystallization.

\section{Acknowledgements}

This work was supported by the NSF (DMR-1310266) and the Harvard MRSEC (DMR-1420570). Part of this work was performed at the Center for Nanoscale Systems (CNS), a member of the National Nanotechnology Infrastructure Network (NNIN), which is supported by the National Science Foundation under NSF award no. ECS-0335765. CNS is part of Harvard University.

\section{Notes and references}

1 R. Laitinen, K. Lobmann, C. J. Strachan, H. Grohganz and T. Rades, Int. J. Pharm., 2013, 453, 65-79.

2 C. Brough and R. O. Williams, III, Int. J. Pharm., 2013, 453, 157-166.

3 Y. Kawabata, K. Wada, M. Nakatani, S. Yamada and S. Onoue, Int. J. Pharm., 2011, 420, 1-10.

4 J. A. Baird, D. Santiago-Quinonez, C. Rinaldi and L. S. Taylor, Pharm. Res., 2012, 29, 271-284.

5 A. Paudel, Z. A. Worku, J. Meeus, S. Guns and G. Van den Mooter, Int. J. Pharm., 2013, 453, 253-284.

6 P. A. Priemel, R. Laitinen, S. Barthold, H. Grohganz, V.-P. Lehto, T. Rades and C. J. Strachan, Int. J. Pharm., 2013, 456, 301-306.

7 Y. C. Ng, Z. Yang, W. J. McAuley and S. Qi, Eur. J. Pharm. Biopharm., 2013, 84, 555-565.

8 E. Amstad, M. Gopinadhan, C. Holtze, C. O. Osuji, M. P. Brenner, F. Spaepen and D. A. Weitz, Science, 2015, 349, 956-960.

9 Y. N. Xia and G. M. Whitesides, Annu. Rev. Mater. Sci., 1998, 28, 153-184.

10 A. Rotem, A. R. Abate, A. S. Utada, V. Van Steijn and D. A. Weitz, Lab Chip, 2012, 12, 4263-4268.

11 D. Law, W. L. Wang, E. A. Schmitt, Y. H. Qiu, S. L. Krill and J. J. Fort, J. Pharm. Sci., 2003, 92, 505-515.

12 K. F. Kelton and A. L. Greer, Nucleation in condensed matter applications in materials and biology, Elsevier, 2010.

13 R. F. Henry, G. Z. Zhang, Y. Gao and I. S. Buckner, Acta Crystallogr., Sect. E: Struct. Rep. Online, 2003, 59, O699-O700.

14 W. K. Burton, N. Cabrera and F. C. Frank, Nature, 1949, 163, 398-399.

15 S. D. Peteves and R. Abbaschian, Metall. Trans. A, 1991, 22, 1271-1286. 